\title{
VISUAL ATTENTION AND ROADWAY LANDMARK IDENTIFICATION IN AT-RISK OLDER DRIVERS
}

\author{
Amy Crowe, Tara Smyser, Mireille Raby, Kirk Bateman and Matthew Rizzo \\ Department of Neurology \\ University of Iowa \\ Iowa City, IA 52242
}

\section{Purpose}

Driving is a complex task that makes high demands on visual processing capacity. Reduction of visual attention and processing speed are risk factors for car crash involvement in older drivers (Owsley et al, 1991; Ball et al, 1993). Impairment of these visual functions can affect driver safety by impairing the ability to search for information in a cluttered panorama that includes traffic signals and road signs. The purpose of the current study was to examine the extent to which on-road landmark identification during the driving task is predicted by off-road measures of visual attention.

\section{Method}

Subjects were 30 drivers, ages 66-92 (mean age=74.6; 57\% male) enrolled in a larger ongoing study of at-risk older drivers. All subjects participated in a battery of tests of visual and cognitive ability. Speed of visual processing, divided attention and selective attention were measured using the Visual Attention Analyzer, 3000 (Visual Resources. Inc., Bowling Green, KY). They also took part in a drive that tested their performance on several attention-related tasks. One of these tasks, a landmark identification task, was developed to evaluate driver perception and attention to relevant stimuli along the roadway. This task required drivers to identify restaurants $(\mathrm{N}=10)$ and safety-related signs (N=7-9) while driving an experimental vehicle along a commercial segment of a four-lane divided state highway. The specific signs to be identified depended on the direction that the subjects drove on the highway. Performance on the landmark identification task was scored in terms of percent correct. Spearman correlation coefficients were calculated between the attention scores and the percent correct scores on the Landmark Recognition task.

\section{Results}

The 30 drivers generally showed impaired performances on tasks of visual processing speed (range $=16-100$, median=16, mean=24.73, $\mathrm{SD}=19.80$ ), divided attention speed (range=23-322, median=86.5, mean=110.67, $\mathrm{SD}=78.61$ ), and selective attention with identification of a foveal target (range $=356-500$, median $=500$, mean $=459.43$, SD=57.40), and with same/different foveal discrimination (range=476-500, median=500, mean=498.87, SD=4.69). Results showed $76.35 \%$ identification for safety signs and $40.3 \%$ correct performance for restaurant identification. The same/different foveal discrimination sub-test showed a significant correlation $(p=0.034)$ with performance on the restaurant portion of the landmark identification task, yet, surprisingly, other relationships were not significant. Participants familiarity with the area did not make a significant difference in their scores $(\mathrm{F}=0.04, \mathrm{p}=0.674)$. Traffic circumstances allowed two drivers extra opportunity to identify signs while stopped at a traffic signal, but this had no significant effect on the results. 


\section{Conclusions}

This study shows that scores on a test of selective visual attention correlated with ability to identify landmarks (restaurants) on a commercial highway, yet visual processing speed and divided attention showed no significant correlation. Moreover, ability to identify safety signs did not correlate with any of the visual measures. Several factors may have affected the percentage of landmarks (signs and restaurants) identified. One factor is the variability of ambient traffic, which causes a variation of driver workload, which in turn affects the ability to search the roadway for visual targets. Also some drivers tended to call out indiscriminately every landmark they encountered, which would tend to increase their percent correct responses. In future analyses we will apply the theory of signal detection to address this response bias.

\section{References}

Ball, K., Owsley, C., Sloane, M.E., Roenker, D.L., Bruni, J.R. Visual attention as a predictor of vehicle crashes in older drivers. Investigative Ophthalmology: Visual Science, 1993; 34:3110-3123.

Owsley, C., Ball, K., Sloane, M.E., Roenker, D.L., Bruni, J.R. Visual cognitive correlates of vehicle accidents in older drivers. Psychology and Aging, 1991;6:403-415. 